\title{
Origanum majorana Essential Oil Reduces VAS Score and Modulates Cerebral Activity during 10 NeurOptimal ${ }^{\circledR}$ Sessions in Patients
}

\author{
María Eugenia Cabaña-Muñoz ${ }^{1 *}$, Carmen Pérez Laso ${ }^{2}$, José María Parmigiani-Izquierdo $^{3}$, \\ José Joaquín Merino ${ }^{4}$
}

CIROM Center (Centro de Implantologia y Rehabilitación Oral Multidisciplinaria; Murcia, Spain)

\author{
${ }^{1,3,4}$ CIROM Center. Murcía (Spain) \\ ${ }^{2}$ Psychobiology Dept. UNED (Madrid, Spain) \\ ${ }^{1}$ mecjj[at]clinicacirom.com \\ ${ }^{4}$ josem2005[at]yahoo.es
}

Running Title: Origanum majorana and NeurOptimal ${ }^{\circledR}$ training

\begin{abstract}
We have evaluated whether NeurOptimal ${ }^{\circledR}$ training could modulate brain activities at pre (PRE) and post-training (POST) during 10 NeurOptimal ${ }^{\circledR}$ (NO) sessions in healthy participants with $(n=13)$ and without Origanum majorana $(n=13)$ stimulation. This essential oil has been impregnated on their nasal filters during all training sessions in (n=26, healthy volunteers; 13 participants that were stimulated with Origanum majorana stimulated with this essential oil as compare with 13 participants without exposure to this odor). Divergence (DIV) reflects the efficacy of NO training on cerebral activity in healthy volunteers with//without Origanum majorana essential oil stimulation during all NO learning sessions in a dental clinic. DIV is the difference of total brain activity reached at Prelearning (PRE) minus Post-training (POST) values. As consequence of NO overtraining, Origanum majorana induces fluctuation on brain activity in these healthy participants. In fact, higher divergences suggest the information has not been integrated at that specific learning session yet. Conversely, negative divergences or values close to zero indicate the information is being integrated at certain moment during NO training. In addition, Origanum majorana stimulation reduces VAS (Visual Analog Scale) scores after NeurOptimal ${ }^{\circledR}$ training as compare to participants non expose to this odor. Thus, Origanum majorana essential oil could induce desired changes on cerebral activity and also reduces variations on brain activities.
\end{abstract}

Keywords: NeurOptimal®/Neurofeedback, brain plasticity, neuromodulation, brain stimulation, aromateraphy (Origanum majorana essential oil), nasal filters (activated carbon), stress/anxiety in patients visiting a dental clinic, biological dentistry, dentist

\section{Introduction}

The inhalation of different odors may modulate emotional response in humans $(1,2)$. The potential antioxidant property of Origanum dictamus essential oil has been reported for some constituents as carvacrol (52\%), gamma-terpinene $(8.4 \%)(2)$. Similar terpenoids are detected in the Origanum majorana essential oil variety used in the present study (alpha-terpinene $14.10 \%$, gamma terpinene $14.1 \%$, Cis Tuyhanol 15.2) among other minoritary components. We have studied whether Origanum majorana stimulation could modulate cerebral activities (Divergences, DIV) during 10 NeurOptimal ${ }^{\circledR}(\mathrm{NO})$ training sessions in healthy volunteers $(\mathrm{n}=26)$ with $(\mathrm{n}=13)$ and without $(\mathrm{n}=13)$ Origanum majorana stimulation during their training. The collective recording of neuron on electrical activity in the form of brain waves is electroencephalography (EEG) (3). EEG can be differenciated into delta wave $(0.5 \sim 3 \mathrm{~Hz})$, theta wave $(4 \sim 7$ $\mathrm{Hz}$ ), alpha wave $(8 \sim 12 \mathrm{~Hz})$, SMR (sensorimotor rhythm) wave $(13 \sim 15 \mathrm{~Hz})$, low beta wave $(16 \sim 20 \mathrm{~Hz})$, high beta wave $(21 \sim 40 \mathrm{~Hz})$ depending on each frequency. Neurofeedback is a noninvasive self-training regimen that improves brain function in the prefrontal cortex, cingulate cortex (4) by inducing specific changes on waves (EEG) $(5,6)$. During NeurOptimal ${ }^{\circledR}$ training $(\mathrm{NO})$, all participants are listening a new age piece music (the same for all of them) given its beneficial effect on physiological parameters (7-11). NeurOptimal ${ }^{\circledR}$ use "music interruptions" to generate changes on brain activity (www.zengar.com, Zengar Institute, Canada). Local and inhibitory/excitatory interactions shape neuronal representations of sensory, motor, and cognitive variables, and produce local electroencephalographic (EEG) gamma frequency (30-80 $\mathrm{Hz}$ ) oscillations $(12,13)$. Alpha and beta wave activity changes can identify emotional responses in patients (14). A beta wave represents a fast wave and is associated with high arousal, concentration and focused attention after Neurofeedback training in patients (15). As consequence of Neuofeedback training, participants improve their cerebral activity in real time (17-21). In fact, Neurofeedback training can induce beneficial effects on working memory, attention (22) and cognitive function (23-27). Up until now, all Neurofeedback-based research on emotion have been conducted with healthy participants and also patients suffering disabilities (28).

\section{Aim}

To study whether NeurOptimal ${ }^{\circledR}$ (NO) training may modulate cerebral activities (Divergence) during $10 \mathrm{NO}$ sessions in participants Origanum majorana stimulated as compare to volunteers without exposure to this fragrance during NO training. 


\section{Methodology (Materials and Methods)}

\section{Origanum majorana essential oil}

The Origanum majorana organic essential oil (PRANAROM®, Spain) has been directly impregnated on their nasal filters (activated carbon, InspiraHealth ${ }^{\circledR}$ Spain) by dilution at $1 \%$ using neutral essential oil. The major volatile constituents of Origanum majorana are terpenes oils (alpha-terpinene $14.10 \%$, gamma terpinene $14.1 \%$, terpinolene $3.17 \%$, Trans-Thuyanol $3.44 \%$, terpinnene 4oil 23,6, alpha-Terpineol 3.1\%, Cis Tuyhanol 15.27, alpha Therpineil $3.1 \%$, Sabinene $8.27 \%$ and others (less than $0.01 \%$.) according to HPLC analysis. This essential oil is free of pesticides (PRANAROM®, Spain), which is an advantage that allow it use in biological dentistry.

\section{Sample Analysis}

This study has been performmed on 26 healthy volunteers (without pathology) following the Declaration of Helsinky $(\mathrm{n}=13$ participants were stimulated with Origanum majorana and 13 without treatment with this fragance during 10 NO learning sessions). All efforts have been made to protect patient's privacy and anonymity. Clinical dental CIROM Center (Centro de Implantologia y Rehabilitación Oral Multidisciplinaria; Murcia) has been approved and certified by AENOR (Spanish Agency of Quality and Certification, Spain). CIROM has the CERTIFICATE for dentist services; CD-2014-001;\#ER-0569/2014 following UNE-EN ISO 9001: 2008 and UNE 179001-2001 Directive (Spain). All patients included in the present study fulfilled the following criteria:

\section{Inclusion criteria}

We have enrolled 26 healthy participants without pathology. Their average age is 45 years old (range age: $38-47$ ) and their sociocultural status is medium/high. $90 \%$ of them are living in Murcia and $10 \%$ are from Alicante (Spain, Europe). All patients were naive to NeurOptimal ${ }^{\circledR}$ training and they never participated in any Neurofeedback/NeurOptimal ${ }^{\circledR}$ study before. NeurOptimal ${ }^{\circledR}$ sessions were conducted by an experienced NO trainer (MEC) during $10 \mathrm{NO}$ training sessions (approximately 1 hour/learning session; 2 sessions/week). All volunteers have completed their NeurOptimal ${ }^{\circledR}$ training within 85 days.

\section{Exclusion criteria}

We have excluded participants with psychiatric/neurological diseases according to the Diagnostic and Statistical Manual of Mental Disorders (DSM-IV-TR, American Psychiatric Association, 2000). We did not enrolled participants who have metabolic diseases/diabetes or those receiving hormonal replacement therapy or taking regular medication (stimulants, anticonvulsants, antidepressant or psychiatric/bipolar drugs). We also excluded those prescripted with chelators or participants taking supplements with antioxidant properties. They had no history of liver/kidney disease/lupus/autoimmune, disease/tyroid diseases/adrenal disease/Cushing syndrome/tumors or menopause. We have also excluded participants taking alcohol, anxiolytic, benzodiacepines or those who had cardiovascular problems.

\section{NeurOptimal@ System}

How Does NeuroOptima ${ }^{\circledR}$ works? NeurOptimal ${ }^{\circledR}$ monitors the brainwaves and gives subtle cues to the brain when it isn't functioning smoothly.

NeurOptimal ${ }^{\circledR}$ (NO) controls brain activity by using a totally non-invasive protocol (Zengar Institute, Canada; www.zengar.com). NeurOptimal ${ }^{\circledR} \quad(\mathrm{NO}$, a version of Neurofeedback) is a more advanced branch of biofeedback that operates using electroencephalogram EEG analysis. The participant uses visual and auditory information offered by NeurOptimal ${ }^{\circledR}$ to re-organize itself the brain activity. The initial shifts may be unrelated to the patients stated goals, because the brain is in control of the prioritization of the changes. The engineering principle of process control is optimized when the measurements of the data stream are accurate, the feedback to the process is both quick and precise and the process adjustment is done only when statically required to promote a directional jump shift or transformation to the next level of organization. No other neurofeedback systems have used the principle of live process control (Zengar Institute, Canada; www.zengar.com). When the brain is performing fluidly, NeurOptimal ${ }^{\circledR}$ plays music but if the brain's activity begins to become inconsistent or less smooth the music and image (fractals in the screen) are interrupted momentarily (www.zengar.com). The interruption gently cues the brain that it isn’t performing optimally NeurOptimal ${ }^{\circledR}$ software dynamically controls the patient feedback using nonlinear statistics to calculate the precise timing that feedback is given. The system responds to the patient's brain wave information and they responds to the system feedback. All of the learning happens outside the conscious awareness. As the brain begins to operate more efficiently, NeurOptimal ${ }^{\circledR}$ adjusts itself automatically individualizing the training microsecond by microsecond to improve the brain's functioning. The participant must avoid to move large muscles or clenching their teeth since the electrical signal strength needed to move muscles will swamp the brain wave signals. The primary feedback is auditory and the visual feedback is supportive during NeurOptimal ${ }^{\circledR}$ performance.

\section{What does mean divergence?}

NeurOptimal ${ }^{\circledR}(\mathrm{NO})$ training indicates the total electrical activity during each pre/post-training session (wave cycles per second). These brain frequencies range from the lowest and slowest Delta waves, to Theta waves, to Alpha waves, to Beta waves, to the highest and fastest Gamma waves above 38 Hertz. Bandwidths of brain frequencies are associated with different states of consciousness.

NeurOptimal ${ }^{\circledR}$ measures brain activities by the Divergence (DIV) parameter, which is an index of brain efficacy. Divergence is the difference of brain activity found at Pretraining (PRE) minus post-training (POST) values during each NO learning session. Divergence can be positive or negative if cerebral activity at Post-training are higher or lower than their respective pre-training (PRE) sessions, respectively. The brain uses this information and reduces or increases or decreases the activity by NO overtraining. Consequently, Divergence (DIV) can reflect "autoplasticity" and cerebral activation. As the brain begins to operate more efficiently, NeurOptimal ${ }^{\circledR}$ adjusts itself automatically in 


\section{International Journal of Science and Research (IJSR) \\ ISSN (Online): 2319-7064 \\ Index Copernicus Value (2013): 6.14 | Impact Factor (2015): 6.391}

response to the brain's activity, individualizing the training microsecond by microsecond. Thus, NeurOptimal ${ }^{\circledR}$ can adjust the necessary brain activity by repeated training since DIV (Divergence) reflects the ganancy or lack of cerebral activity reached during these $10 \mathrm{NO}$ sessions.

Divergence is a measure of stability. The brain activity at pre\&postraining at CAC graphs are compared and used as a guide to measure progress of training (Fig 1a-d). Divergences reflects the relative stability of the brain. The lower the number the more stable the patient' nervous system is. Progress is not linear, meaning that the divergence numbers do not go down in a straight line orderly fashion. When divergences are higher means that information has not been integrated at that session and the brain is trying to learn the information without success. Conversely, negative divergences suggest the information have been integrated at that particular NO session. However, if cerebral activity at post-training (POST) are close at pre-training (PRE) values or even though higher, the information will be progressively integrated during NO training. The brain uses these fluctuations on cerebral activity to adjust the optimal state.

\section{NeurOptimal® Technology}

How does work NeurOptimal ${ }^{\circ}$ client Hookup?NeurOptimal ${ }^{\circledR}$ Patient Hookup consists of silver electrodes, applied to the clients'ears \& scalp, centered between the ear and the crown of the head on the bony ridge (Central points, cortex C3 \& C4). The electrodes are applied with EEG paste that it is water soluble electrical conductance material composed primarily of salts that enhance the monitoring of the minute electrical pulses of the brain during pre/post-training sessions (from 1 to session$10)$.

Z-amp ${ }^{\text {TM }}$ Amplifies Signals. The electrode sensors pickup the brains electrical signal and send that signal down a conductance wire to the Zengar Z-amp ${ }^{\mathrm{TM}}$ (NeurOptimal ${ }^{\circledR}$ ). This Z-amp ${ }^{\mathrm{TM}}$ cleans line noise and amplifies the brain wave signal. Other neurofeedback data looks "smeared" as compare to NeurOptimal ${ }^{\circledR}$ 's data due to a sampling rate of 256 samples per second, which are coupled with high precision of filtering, targeting and triggering of feedback. At no time are electrical signals fed back to the brain.

Signal Separation into Frequencies and Intensities. The left and right brain wave signals are then separated by the computer software into their component frequencies and intensities.

Non-linear Statistical Analysis of Data. This continuous data set is analyzed over time using non-linear dynamical math and statistics in order to determine when brain and nervous system enter into an area of "unstable" operation and feedback is given instantly within milliseconds. The feedback is given in the form of a pause in the music that is being listening and a momentary hesitation of the fractal image appears in the screen of the computer. This mean that brain disturbances are associated to a pause in the music at pre and post- learning sessions.

Variables of time, frequency and intensity. Feedback is determined by continuously tracking the 3 variables of time, frequency and intensity. This is called Joint time frequency analysis (JTFA).

"Dynamic Dance." The continuous data set is analyzed by the software using 16 different target filters simultaneously. Each of these targets works dynamically with the brain at that exactly time. The result of this "dance" between the participant's brain and the NeurOptimal ${ }^{\circledR}$ software that is broad-based and integrated patient transformation.

Initial learning Session. During the initial session, the Expert NeurOptimal ${ }^{\circledR}$ (MEC) trainer has instructed to all participants. During the upcoming 10 learning sessions, the total brain's frecuencies have been measured at pre (PRE) and post-training (POST) (approximately 1 hour/patient). All participants must be comfortably reclining in a cozy chair with a warm blanket in a darkened room during each learning session. The client has EEG sensors attached and a set of stereo ear phones on. At the end of each session, the pretraining phase (PRE) takes 30 seconds (PRE, 15 seconds with open eyes and 15 seconds with close eyes) and the Post-training phase takes 33 minutes and 30 seconds POST). Inmediately after concluding Pre-training (PRE), have followed the post-training phase without interruption. NO training have been done two times by week (from 8:00 to 14:00 hours A.M and t) and all volunteers have concluded they training within 85 days. Each cerebral activity have been measured at PRE/POST, DIV (divergences) in those volunteers with $(\mathrm{n}=13) /$ without $(\mathrm{n}=13)$ Origanum majorana stimulation during $10 \mathrm{NO}$ sessions. The essential oil has been impregnated at $1 \%$ on their nasal filter during all training sessions (from PRE to POST, 10 sessions). Participants without Origanum majorana stimulation did not smell this essential oil during all NO training sesisons in dental clinic (CIROM)

\section{Pre Baseline Graphical Results and PRE/POST learning sessions in NeurOptimal ${ }^{\circledR}$.}

The pre learning phase indicates the baseline for cerebral activity during 30 seconds of PRE-training. After each NO session, the system shows a spectrograph image and the CAC graph (Cross correlation of the auto correlation on right) after post-training in order to indicate the progression of training on cerebral activities. The baselines line takes 30 second during prelearning (PRE) and indicates snapshot of NO performance.

The Training Period. During each Pre-learning session (PRE), the music is listened during 30 seconds and $33 \mathrm{~min}$ at post-training (POST). Each participant will listen to NeurOptimal ${ }^{\circledR}$ soothing instrumental new age music (the same piece for all participants). They will also be watching a monitor with a kaleidoscope of engaging fractals images. The optimal length of NeuroFeedback training series seen to be close to twenty sessions in patients with disabilities (16) although efficacy of NO training are expected to occur in six sessions. In our study, NeurOptimal ${ }^{\circledR}$ performance takes 10 learning sessions ( 2 times/week) to assure that the changes are correctly established in participants. Anyway, over NeurOptimal ${ }^{\circledR}$ training is not a problem if are necessary more learning sessions. Usually there will be noticeable shifts within 6 sessions. 


\section{International Journal of Science and Research (IJSR) \\ ISSN (Online): 2319-7064 \\ Index Copernicus Value (2013): 6.14 | Impact Factor (2015): 6.391}

Patient Given Feedback. Graphical Displays of Brain Wave Variation appears during the training period and many different displays (Spectra left and Helix right) represent the minute by minute unfolding of the patient brain wave activity. The brain activity intensity will trigger the various frequency target filters as the training unfolds. When the nervous system statistically enters into an unstable operating area, feedback is given very rapidly, with precise timing, within milliseconds. The feedback is given in the form of a very brief pause in the music and a momentary hesitation on the fractal image, which means that some brain disturbances could occur exactly at that moment. Sometimes the pauses are so quick you may not consciously notice them. "This pause is quite literally the pause that refreshes". A pattern of pauses are given to the brain. The brain immediately recognizes that these patterns are important and begins to self reorganizates. "When it comes to the frequency of feedback, LESS IS MORE". This mean that divergences drops over cero (Negative divergences). Consequently, the brain activity is progressively decreasing after post-training. These divergence identify when brain activities are increasing and downs in order to reach a better brain reorganization state in patients. The large drop in divergence numbers from before the training session to after indicates that brain is working hard to shift into regulation to finally reach a calm state (divergences close to zero or even though negatives). The NeurOptimal ${ }^{\circledR}$ software automatically has four different modes called Zen 1,2,3,4,5 (see below; i.e: Zen 5 version mode of 45 minutes). The current protocol of 33 minutes and 30 seconds have been used in the present study.

\begin{tabular}{|c|c|c|c|c|c|}
\hline Session & Zen 1 & Zen 2 & Zen 3 & Zen 4 & Total Time \\
\hline Demo & 5 & 5 & & 5 & 15 \\
\hline First & 11 & 11 & & 11.5 & 33.5 \\
\hline Second & 8 & 12 & 5 & 8.5 & 33.5 \\
\hline Third & 7 & 8 & 10 & 8.5 & 33.5 \\
\hline Regular & 5 & 7 & 14 & 7.5 & 33.5 \\
\hline Extended & 5 & 10 & 20 & 10 & 45.0 \\
\hline
\end{tabular}

Modes of NeurOptimal ${ }^{\circledR}$ learning are indicated as

\section{follows:}

\begin{tabular}{|l|c|c|}
\hline Modes & Exercise & Difference between Modes \\
\hline Zen 1 & Warm up & $\begin{array}{c}\text { Both sides of the brain are trained separately } \\
\text { over all frequencies, no comparison between } \\
\text { sides }\end{array}$ \\
\hline Zen 2 & Weight lift & $\begin{array}{c}\text { Both sides of the brain are trained together } \\
\text { within each separate target range }\end{array}$ \\
\hline Zen 3 & Endurance & $\begin{array}{c}\text { Both sides of the brain are trained together } \\
\text { over all frequencies at the same time }\end{array}$ \\
\hline Zen 4 & Cool down & Integration of learning \\
\hline
\end{tabular}

\section{Statistical Analysis}

Data were analysed by using SPSS software (V17.0), and Sigma Plot (11.0). Mean and standard deviation were estimated during each Pre and Post-training (10 sessions). [from initial session $1(\mathrm{~S}-1)$ to the last one: session 10 (S10)]. All variable were expressed as mean values + - relative error (standard deviation divided by $n$ root), being $n$ the size sample $(n=26)$. The effectiveness of NeurOptimal ${ }^{\circledR}$ training were evaluated by a repeated analysis of variance (ANOVA) for divergence (DIV), PRE, or POST-training values, including session (S1 to S10) as the repeated factor, group as the between-groups factor and cerebral activities at Pre, Post-training and Divergences (DIV) as dependent variables. Data were expressed as mean+-relative error betweengroups. The pos Hoc t Student or Mann Withney test have analyzed possible significant differences between volunteers with/without Origanum majorana stimulation depending if there were homogeneity of varianze or not, respectively. The W Machutly indicates sphericity and Greenhouse-Geisser shows significant differences $(\mathrm{p}<0.05)$. Data were compared using multiple comparisons, where $p$ values of 0,05 or less were considered significant.

\section{Results}

The repeated ANOVA indicates a strong tendency to reach a significant effect for total Divergence (positive and negative) $[\mathrm{F}=3 ., 19, \mathrm{p}=0.091 ; \mathrm{n} . \mathrm{s}$, Etha square $=0,15$, power $=0,4$ ) according to Greenhouse-Geisser data (Figure 1c). The Pillai test for multivariant contrast indicates a significant effect for total Divergence (DIV) $[\mathrm{F}=3,17$, $\mathrm{p}=0,043$, Beta $=0.74])$.

Fig 1a. Effects of NeurOptimal ${ }^{\circledR}$ training on brain activities at PRE-training in healthy volunteers with/without Origanum majorana stimulation during 10 sessions.

The repeated ANOVA shows a significant effect on cerebral activities at PRE-learning $[\mathrm{F}=76, \mathrm{p}<0,05$, Etha square $=0,69$, power $=0,7$ ] according to Greenhouse-Geisser data. The Pillai test for multivariant contrast reveales a significant effect on cerebral activities at PRE-learning $[\mathrm{F}=3,83$, $\mathrm{p}<0,05$, Etha square $=0,69$, power $=0,85)$. W Mauchly indicates sphericity $\mathrm{W}=0,000, \mathrm{X}$ Square $=195,1 ; \mathrm{p}<0.05$ ).

Origanum majorana treatment induces significant changes (t-test or Mann Withney) on brain activities at certain pretraining sessions (PRE) in healthy volunteers (Session-3 (S3), S6 and S7) as compare to their respective session during training in participants without stimulation with this fragrance $(p<0.05$, Figure 1a).

Fig 1b. Effect of NeurOptimal ${ }^{\circledR}$ post-training (POST) training on cerebral activities in healthy volunteers with/without Origanum majorana during 10 sessions. The repeated ANOVA reveales that NeurOptimal ${ }^{\circledR}$ postraining (POST) trend to increase cerebral activities as consequence of progressive NO training $[\mathrm{F}=2,04, \mathrm{p}<0,13$; n.s, power $=0,44)$ ] according to Greenhouse-Geisser data. The Pillai test for multivariant contrast indicates a significant change on cerebral activities during POSTtraining sessions $[\mathrm{F}=3,83, \mathrm{p}<0,05$, Etha square $=0,6$, power $=0,85]$. Pos hoc analysis reveales that Origanum majorana $(\mathrm{n}=13)$ stimulation induces significant increases on cerebral activities at certain POST-training sessions (ttest or Mann Withney, Session-4: S4, S7 and S9) as compare to their respective sessions in healthy participants $(n=13)$ non stimulated with this essential oil ( $p<0.05$, Figure $1 b)$.

Fig 1 c. Effect of NO training on total cerebral activities (Divergence) in healthy participants with/without Origanum majorana stimulation. 


\section{International Journal of Science and Research (IJSR) \\ ISSN (Online): 2319-7064 \\ Index Copernicus Value (2013): 6.14 | Impact Factor (2015): 6.391}

The repeated ANOVA reveales a trend to modulate Divergence in healthy volunteers with/without Origanum majorana stimulation during $\mathrm{NO}$ training $[\mathrm{F}=3,19 ; \mathrm{p}=0,091$; n.s; Etha partial $=0,15$; Beta $=0,4]$. Interestingly, MannWithney or t Student post Hoc analysis reveales raises on total cerebral activity (positive and negative) in participants stimulated with Origanum majorana essential oil at certain NO sessions (S4 and S8) as compare to participants without treatment with this odor (Figure $1 \mathrm{c}, \mathrm{p}<0.05$ ).
Fig 1d. Origanum majorana modulates negative cerebral activities (Negative divergences, DIV (-) during 10 NeurOptimal ${ }^{\circledR}$ sessions.

Interestingly, Origanum majorana significantly affects negative divergence (DIV) at certain sessions (S3 and S6) as compare to volunteers non stimulated with this fragrance during learning $(\mathrm{p}<0.05)$.

Fig 1a

Effect of Origanum majorana on cerebral activity at PRE-training

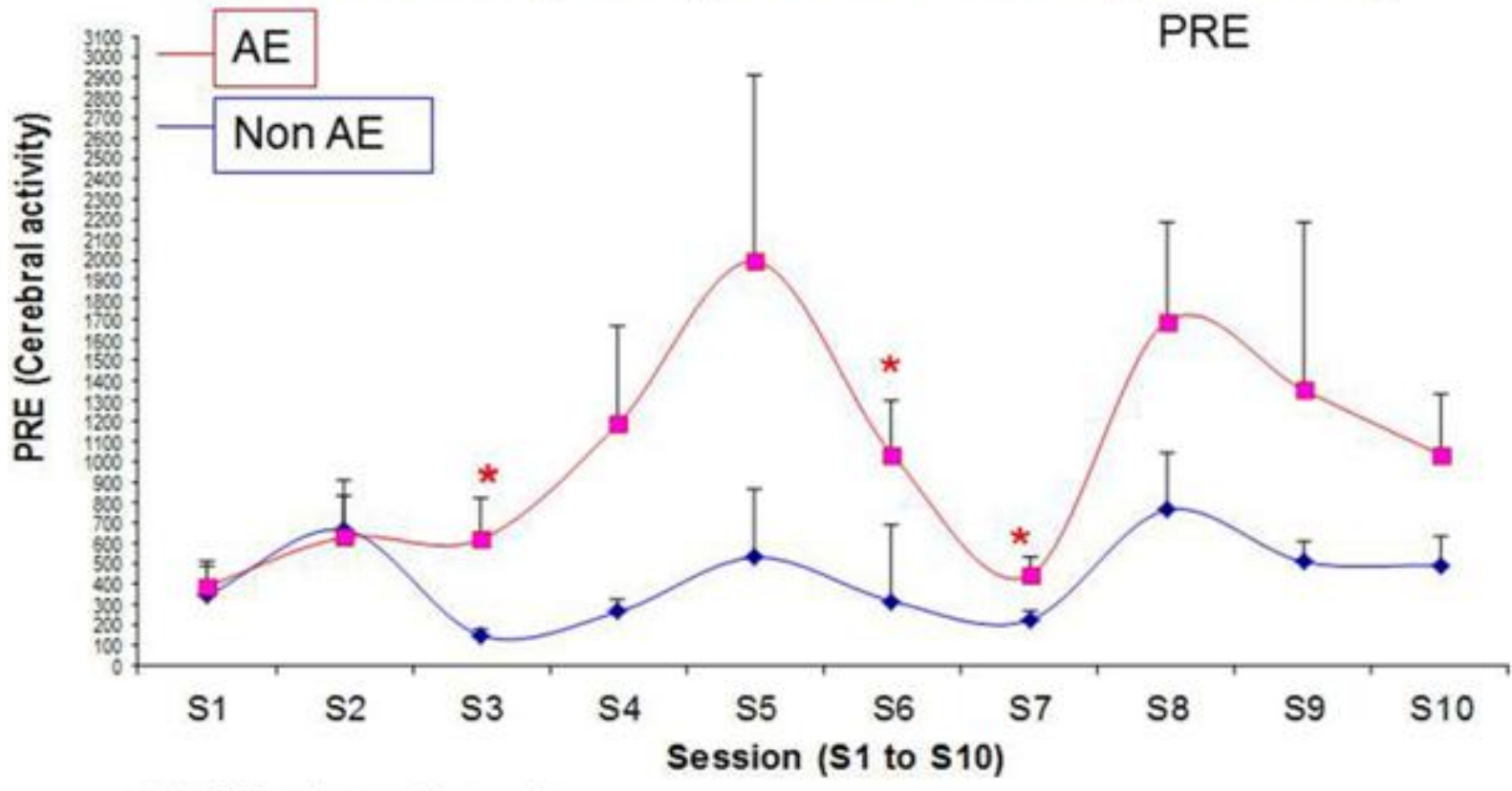

${ }^{2} p<0.05$ vs its respective session

Fig 1b Effect of Origanum majorana on cerebral activities at POST-training

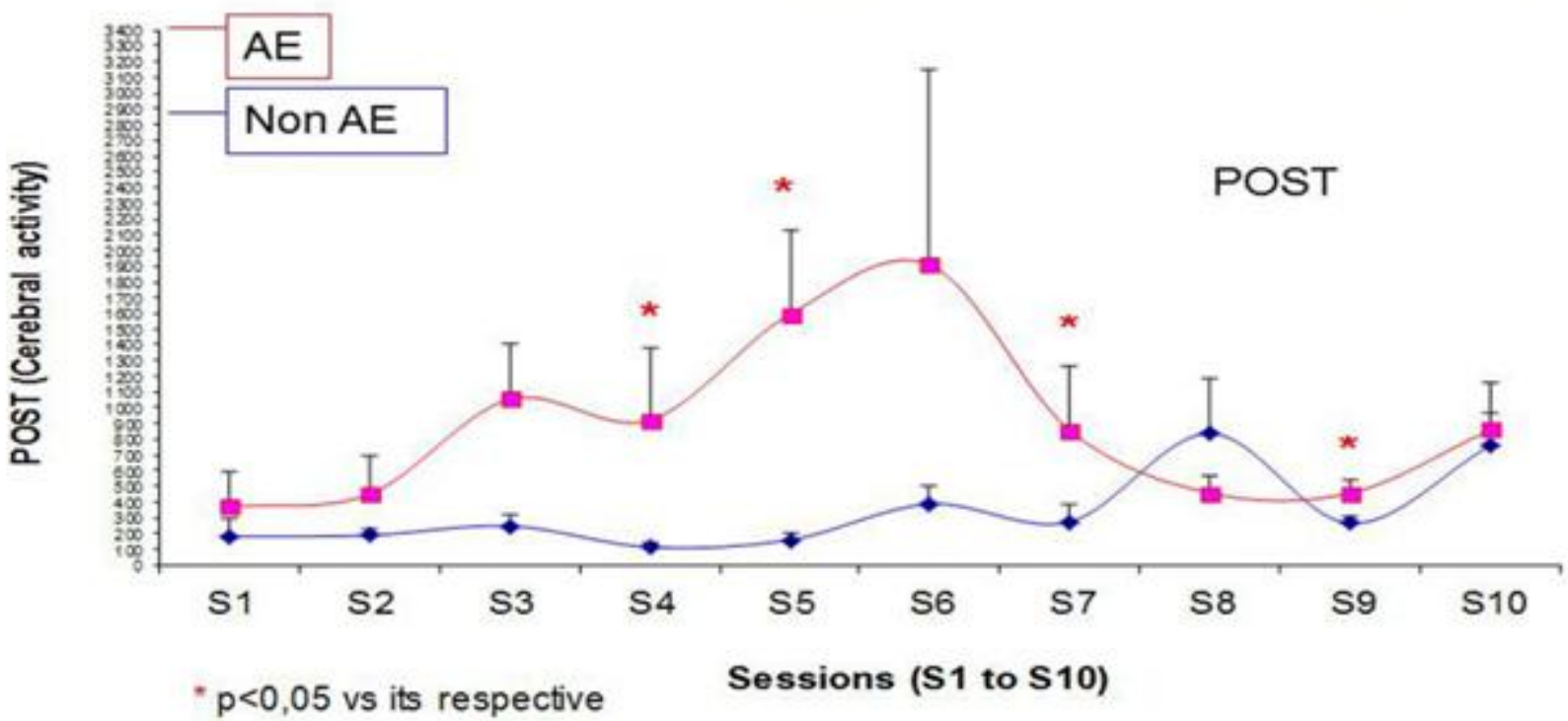

Volume 5 Issue 7, July 2016 www.ijsr.net 
Fig 1c

Effects of Origanum majorana on total cerebral activities (DIV)

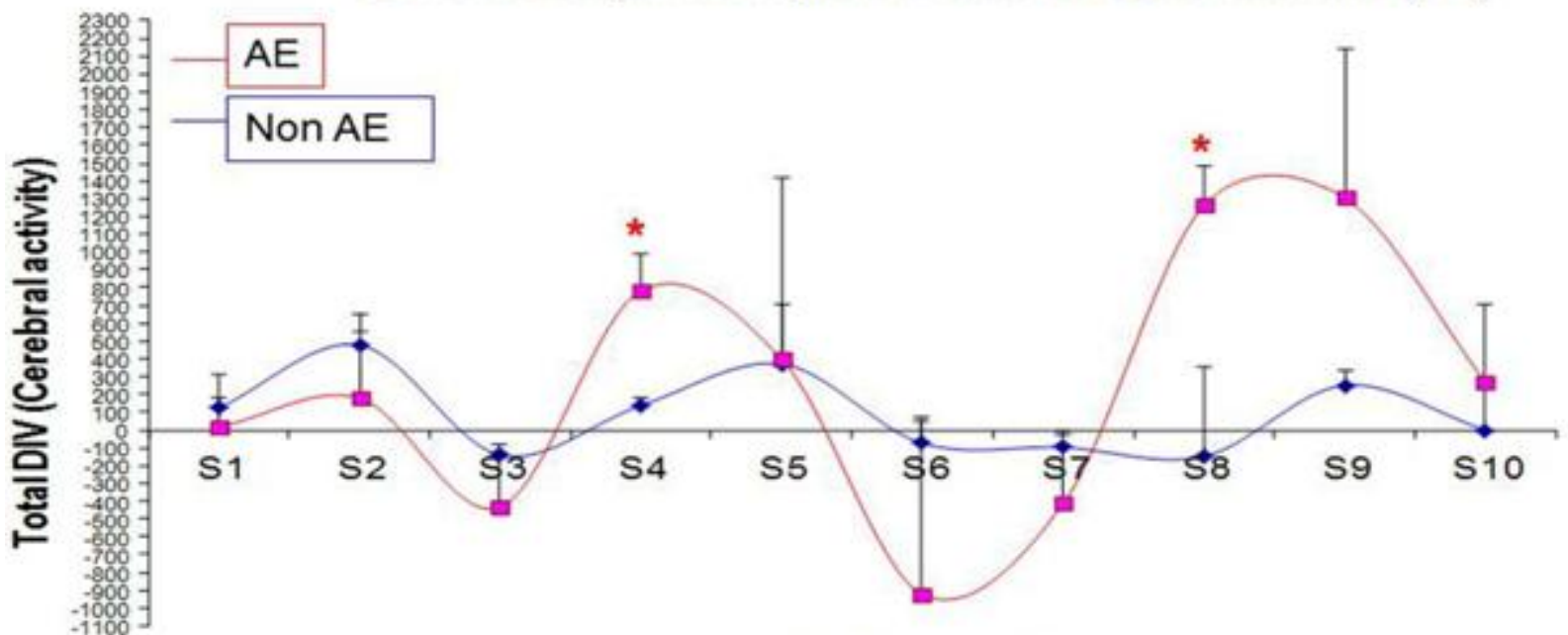

Sessions ( 1 to $\mathrm{S} 10)$

- $p<0,05$ its respective session

Fig 1d

Effect of Origanummejorana on Negative Divergence

Session (S1 to S10)

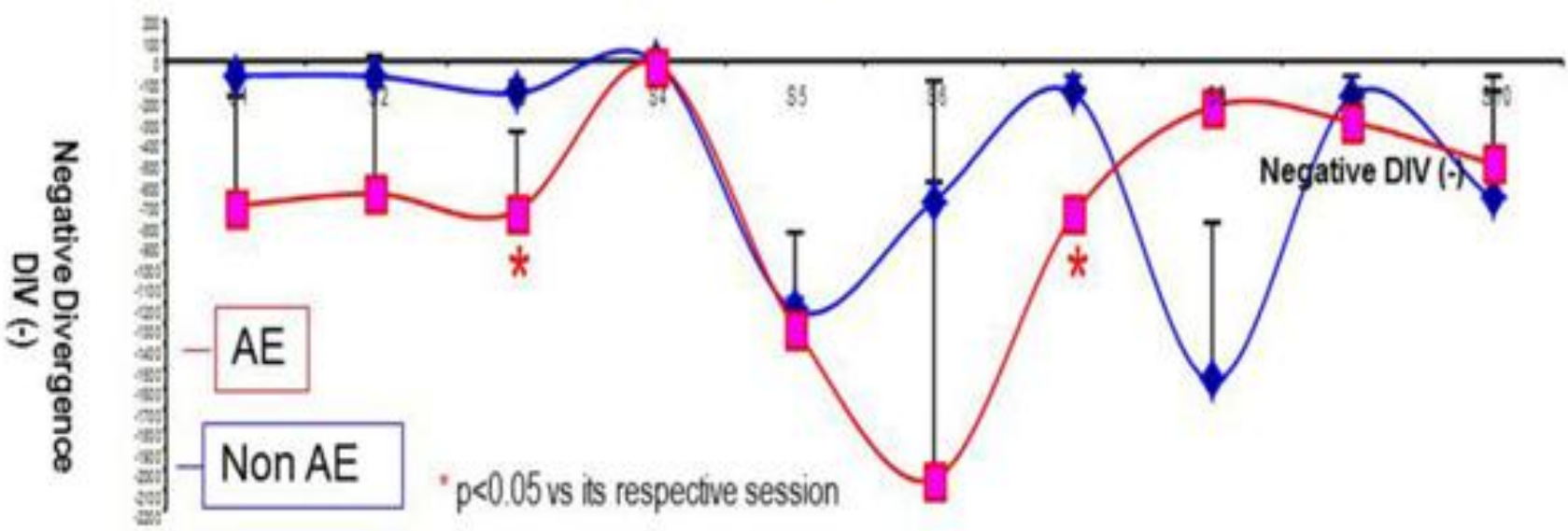

Figure 1 legend

Fig-1a and 1b. Effect of NeurOptimal ${ }^{\circledR}$ training on cerebral activities at Pre-learning in healthy participants stimulated with Origanum majoranam $(n=13) /$ without $(n=13)$ treatment with this essential oil during $10 \mathrm{NO}$ sessions

$\mathrm{PRE}=$ Brain activity at prelearning (Fig 1a, 260 measurements).

PRE-AE $=$ Brain activity at prelearning in healthy participants (Fig 1a) in Origanum majorana stimulated participants during NeurOptimal ${ }^{\circledR}$ training.

POST=Brain activities at post-training (260 measurements) POST-AE=Brain activities in healthy participants after Origanum majorana treatment during 10 NeurOptimal ${ }^{\circledR}$ learning sessions (Fig 1b).

Fig 1c. Total divergences (positive and negative) observed in healthy participants with $(n=13,260$ data) and without
Origanum majorana stimulation during 10 NeurOptimal ${ }^{\circledR}$ sessions (n=13, 260 data). The Fig 1d indicates negative divergences during NeurOptimal ${ }^{\circledR}$ sessions (NO) only. Total DIV is the cerebral activity found at Prelearning (PRE) minus POST-training(POST) values in $10 \mathrm{NO}$ sessions.

Red color: participants stimulated with Origanum mejorana during 10 NO learning sessions (AE).

Blue color: participants that did not smell this fragrance during 10 NO learning sessions (Non AE)

The figure 2 a,b,c show representative case of moderate (Fig 2a), low Divergence (Fig 2 b,c) after NeurOptimal ${ }^{\circledR}$ performance in healthy volunteers (PRE-yellow color; POST-orange color) 
International Journal of Science and Research (IJSR)

ISSN (Online): 2319-7064

Index Copernicus Value (2013): 6.14 | Impact Factor (2015): 6.391
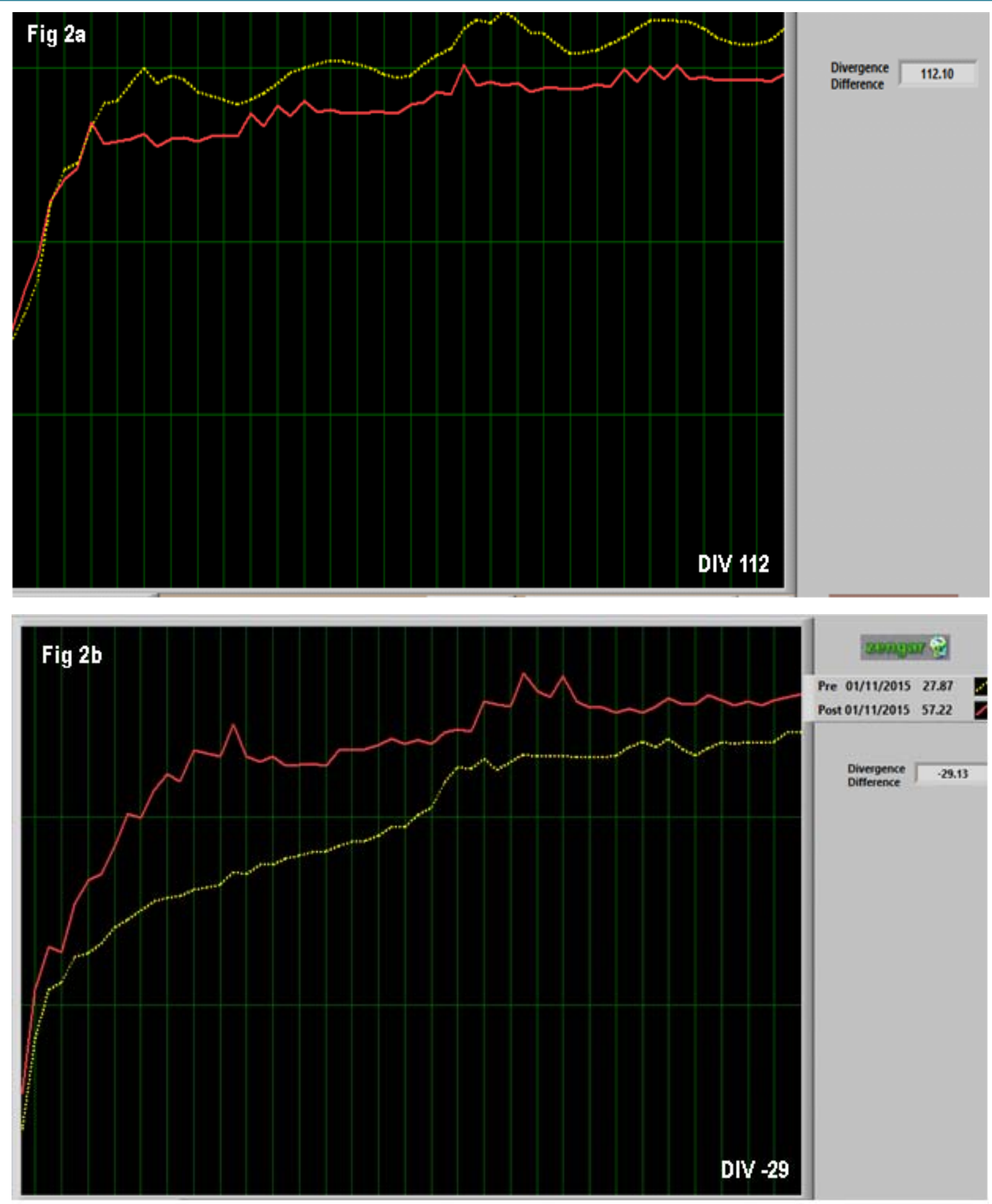

Volume 5 Issue 7, July 2016 www.ijsr.net

Licensed Under Creative Commons Attribution CC BY 


\section{International Journal of Science and Research (IJSR) \\ ISSN (Online): 2319-7064}

Index Copernicus Value (2013): 6.14 | Impact Factor (2015): 6.391

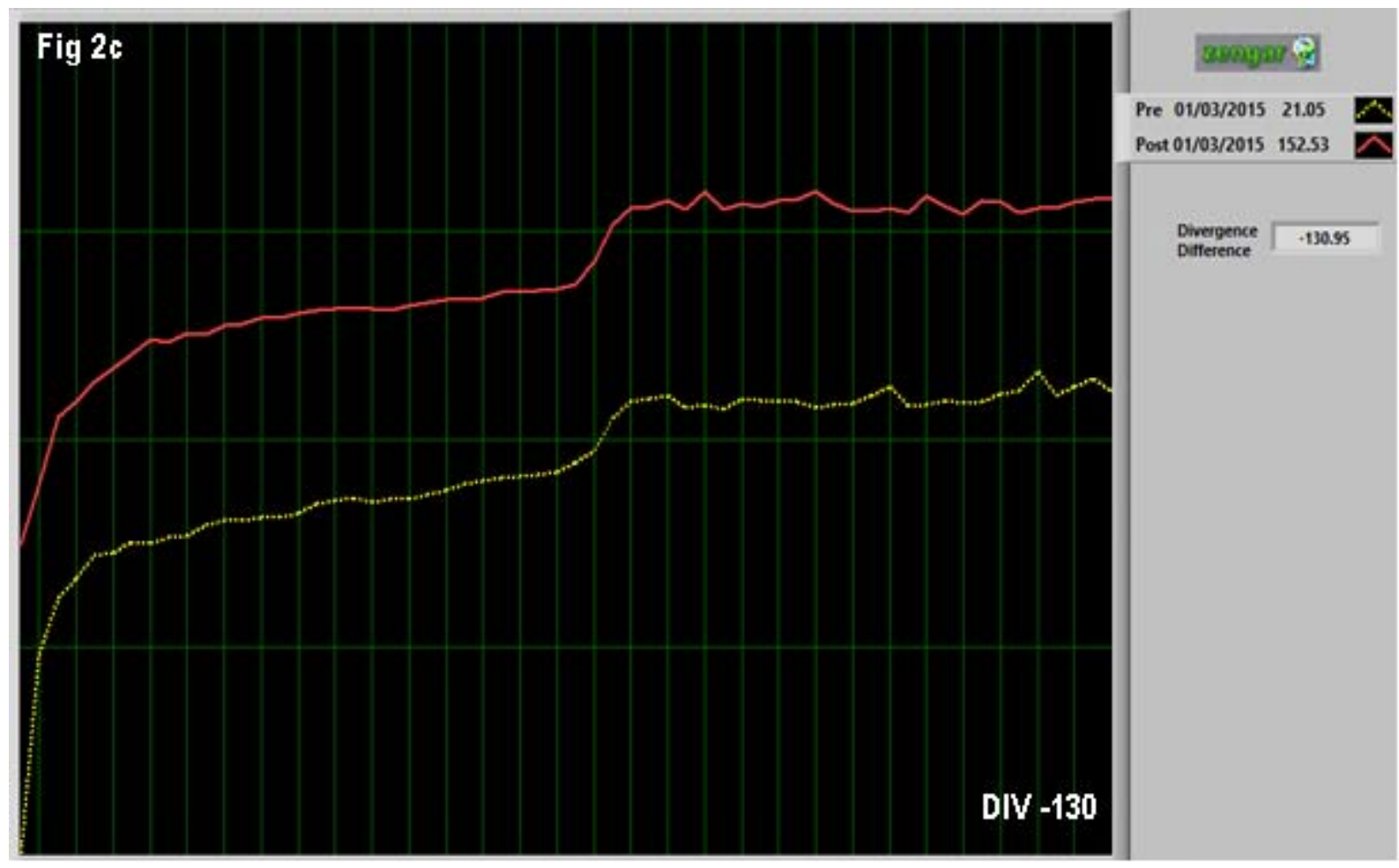

Fig 3. Origanum majorana essential oil improves Visual Analog score (VAS, $65 \%$ ) in healthy volunteers as compare to participants non stimulated with this odor.

After 10 NO learning sessions, healthy participants that were Origanum majorana stimulated experienced $65 \%$ of improvement on VAS score (percentage of improvement) (Figure 3 ) after 10 NeurOptimal ${ }^{\circledR}$ learning sessions.

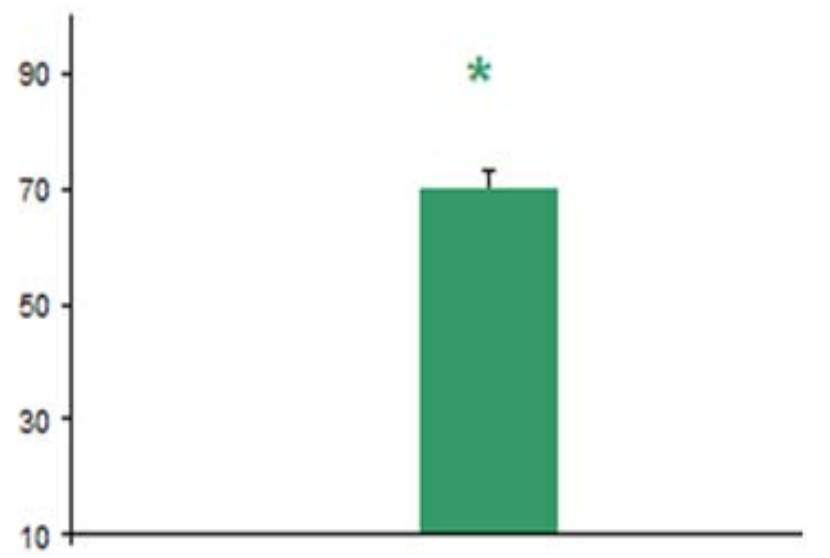

${ }^{*} \mathrm{p}<0.05$ as compare to participants without Origanum majorana stimulation during NO training.

Figure 3. NeurOptimal ${ }^{\circledR}$ training improves VAS score $(65$ $\%$ ) after Origanum majorana treatment in healthy volunteers as compare to participants without exposure to this essential oil.

$\mathrm{n}=26$ healthy volunteers $(\mathrm{n}=13$ with Origanum majorana essential oil stimulation, $\mathrm{n}=13$ without Origanum majorana treatment during $10 \mathrm{NeurOptimal}{ }^{\circledR}$ sessions).

\section{Discussion}

It is known that Neurofeedback training provides an effective way to train electrophysiological activity during training in patients $(15,26,27)$. This is the first study showing that Origanum majorana essential oil stimulation in participants significantly reduced Visual Analog Scale scores (VAS: $65 \%$ of improvement) after 10 NeurOptimal ${ }^{\circledR}$ (NO) training sessions. In addition, healthy participants displayed fluctuations on cerebral activity after Origanum majorana essential oil stimulation by impregnation on their nasal filter during all training sessions. The inhalation of fragances or essential oils could influence the reaction time in humans under pleasant and non-pleasant exposure to odors (28). Our findings suggest that NO training is a safe and non invasive alternative that can improve learning abilities and also can reduce stress/anxiety in patients visiting a dental clinic $(28,30,32)$. However, NO training may also induce fluctuations on cerebral activity by enhancing desired electro-cortical activity and could suppress undesirable activity at certain moments in our study. Interestingly, Origanum majorana induces significant raises on brain activities at session 4 and $8(\mathrm{~S}-8)$. This effect could be necessary to modulate undesired electro-cortical activity by inducing moderate increase on divergence at certain moments. The lateralizations of brain activation for pleasant odors have been reported in right hemisphere whereas unpleaseaset smells were activated on lelf hemisphere (29). In our study, all participants had cortical electrodes on both hemisphere.

The volatile constituent from Origanum majorana essential oil (terpenoids) may enter the bloodstream since this odor was directly impregnated on their nasal filter. These patients experienced lower VAS score after Origanum majorana stimulation during training sessions. These findings agree with data showing that Rosmarinus officinal (30) or Lavander eseential oil exposure induce beneficial effects in

\section{Volume 5 Issue 7, July 2016}




\section{International Journal of Science and Research (IJSR) \\ ISSN (Online): 2319-7064 \\ Index Copernicus Value (2013): 6.14 | Impact Factor (2015): 6.391}

healthy patients when they were expose to these fragance. However, these odors were not impregnated on nasal filters in these related studies (31-33). The lavander experimental group reported lower current anxiety in a dental clinic (32), which agree with our findings since Origanum majorana has been impregnated on their nasal filters during training here. These terpenoids from Origanum majorana essential oil could diffuse through the olfactory bulb and pass to the limbic system. In fact, aromateraphy can stimulate the olfactory bulb (34) in neuroimage studies (35). Several terpenoids from Origanum majorana essential oil may modulate cerebral activities as consequence of NO ovetraining here. However, aromateraphy also induces fluctuations on brain activities (Divergences) at certain learning sessions (S4 and S8), suggesting that are necessary peaks on cerebral activity before reaching a better brain adaptations states as consequence of repeated NO training. When divergence (DIV) reachs negative values at posttraining (POST) or are close to pre-learning values, the information would be integrated by progressive NO overtraining (10 sessions, Figure 1a-d). If divergences (DIV) became more negative, the brain is being quickly "reorganizate" and progressively recognizes and felts the NO feedback is important (Fig 1c,d). The stimulation of olfactory bulb may improve cognitive abilities, also in healthy participants (36). In fact, terpenoids from Origanum majorana were directly impregnated on their nasal filters during NO training. These findings support the synergic use of Origanum majorana essential oil together NeurOptimal ${ }^{\circledR}$ training in order to reduce stress or ameliorate bruxim in patients visiting a dental. Although our study can not elucidate the activated brain areas by NO training, some Neurofeedback studies have demonstrated significant changes on brain connectivity in dorsomedial prefrontal cortex and amygdala after learning. After Neurofeedback training, participants successfully self-regulated the topdown connectivity within these brain areas $(13,36)$. Our findings support that NO technology is a safe alternative to improve physchological function and also can enhance cognitive abilities in patients $(13,36)$. In addition, the use of these nasal filters (activated carbon) could reduce exposure to heavy metals during dental treatments in biological dentistry $(37,38)$. Our findings suggest the these nasal filter may vehiculizate essential oils with therapeutic properties to reduce stress/anxiety in patients visiting a dental clinic (37).

\section{Conclusion}

Origanum majorana essential oil induces fluctuation on brain activities as consequence of repeated NeurOptimal ${ }^{\circledR}$ training (10 sessions) in healthy participants. The NeurOptimal ${ }^{\circledR}$ ("a version of Neurofeedback") technology is a safe protocol that modulates cerebral activities during 10 NO training sessions. In fact, Origanum majorana stimulation during NeurOptimal ${ }^{\circledR}$ training reduced VAS scores during 10 sessions. However, fluctuations on brain activities could promote desired changes on electro-cortical activity and supress undesirable changes at certain NO sessions. Interestingly, Origanum majorana stimulation increases brain activities at certain sessions 4 and 8 (S-4, S8); This feature suggest that brain activities fluctuate (DIV: Divergences) before getting a better brain reorganization state in these trained participants. Collectively, Origanum majorana (aromatherapy) essential oil stimulated positively modulates the alertness states, which agree with some beneficial described effects of aromateraphy on EEG activity (36). Finaly, these nasal filters can vehiculizate essential oils with therapeutic properties in patients visiting a dental clinic.

\section{Conflict of Interest}

None to declare

All authors declare no conflict of interest.

\section{Author's Contribution}

All authors have substantial contributed to the conception/design, data acquisition interpretation, and discussion.

Wrote the paper: JJM, MEC.

Conceived and designed the study: MEC, CPL, JMP, JJM

Performed the experiments: JJM, JMP, MEC

Statistical analysis of data: JJM, JMP, CPL, MEC

\section{Acknowledgements}

We would like thank all enrolled volunteers for their collaboration. Findings supported by PRANAROM ${ }^{\circledR}$ (Barcelona, Spain). We thank Roberto Pierobon, Ana Roselló and CIROM research team for their important colaboration (Murcia). MEC, an advanced Neurofeedback trainer, thank Samantha Diavatis (Zenger Institute, Canada) her advice on NeurOptimal ${ }^{\circledR}$ technology.

The Origanum majorana essential oil was supplied by PRANAROM ${ }^{\circledR}$ (Spain). http://www.pranarom.com/es/home

We also thank InspiraHealth ${ }^{\circledR}$ (Spain) the supply of nasal filter. http://www.inspirahealth.es/

\section{References}

[1] Moss,M.,Cook, J.,Wesnes, K.,Duckett, P.Aromas of rosemary and lavender essential oils differentially affect cognition and mood in healthy adults. Int $J$ Neurosci.2003;113(1): 15-38.

[2] MitropoulouG, Fitsiou E, Stavropoulou E, Papavassilopoulou E, Vamvakias M, Pappa A, Oreopoulou A, Kourkoutas Y. Composition, antimicrobial, antioxidant, and antiproliferative activity of Origanum dictamnus (dittany) essential oil.Microb Ecol Health Dis.2015;6(26): 26543.

[3] Chanel, G., Kronegg, J., Grandjean, D., Pun T.Emotion assessment: arousal evaluation using eeg's and peripheral physiological signals, inMRCS 2006. LNCS,Vol. 4105, eds Gunsel B., Jain A. K., Tekalp A. M., Sankur B., editors. (Heidelberg: Springer. 2016: 530-537.

[4] Koush, Y., Meskaldji, D.E., Pichon, S., Rey, G., Rieger, S.W., Linden, D.E, Van De Ville, D., Vuilleumier, P., Scharnowski, F. Learning Control Over Emotion Networks Through Connectivity-Based Neurofeedback. Cereb Cortex. 2015;17. 


\section{International Journal of Science and Research (IJSR) ISSN (Online): 2319-7064}

Index Copernicus Value (2013): 6.14 | Impact Factor (2015): 6.391

[5] Cohen Kadosh,K., Linden, D.E, Lau, J.Y. Plasticity during childhood and adolescence: innovative approaches to investigating neurocognitive development. Dev Sci. 2013;16(4): 574-83.

[6] Tallon-Baudry, C., Bertrand, O., Delpuech, C., Pernier, J. Stimulus specificity of phase-locked and non-phaselocked $40 \mathrm{~Hz}$ visual responses in human. J Neurosci.1996;16(13): 4240-9.

[7] Lundqvist, L.O., Carlsson, F., Hilmersson, P., Juslin, P.N.Emotional responses to music: experience, expression, and physiology. Psychol. Music 2009; 37: 61-90.

[8] Benz, S., Sellaro, R., Hommel, B., Colzato, LS.Music Makes the World Go Round: The Impact of Musical Training on Non-musical Cognitive Functions-A Review. Front Psychol. 2016;7, 6: 2023.

[9] Choppin, S., Trost, W., Dondaine, T., Millet, B., Drapier, D., Vérin, M., Robert, G., Grandjean D. Alteration of complex negative emotions induced by musicin euthymic patients with bipolar disorder. J Affect Disord. 2016;191: 15-23.

[10] Särkämö, T., Laitinen, S., Numminen, A., Kurki, M., Johnson, J.K., Rantanen, P. Pattern of Emotional Benefits Induced by Regular Singing and Music Listening in Dementia. J Am Geriatr Soc. 2016; 64(2): 439-40.

[11]Zhao, K., Bai, Z.G., Bo, A., Chi, I. A systematic review and meta-analysis of music therapy for the older adults with depression. Int J Geriatr Psychiatry. 2016; 19.

[12] James, C.J., Wang, S. Blind source separation in single channel EEG analysis: an application to BCI.Conf Proc IEEE Eng Med Biol Soc.2006;Suppl:6544-7.

[13] Coben,R., Myers,T.E. The relative efficacy of connectivity guided and symptom based EEG biofeedback for autistic disorders. Appl Psychophysiol Biofeedback. 2010; 35(1): 13-23.

[14] Ramirez, R., Vamvakousis, Z. Detecting emotion from EEG signals using the emotive EPOC device in Proceedings of the 2012. International Conference on Brain Informatics, LNCS 7670 (Macau: Springer). 2012;175-184.

[15] Auer, T., Schweizer, R., Frahm, J. Training Efficiency and Transfer Success in an Extended Real-Time Functional MRI Neurofeedback Training of the Somatomotor Cortex of Healthy Subjects. Front Hum Neurosci. 2015; 9(9): 547.

[16] Simoes M, Lima J, Direito B, Castelhano J, Ferreira C, Carvalho P, Castelo-Branco M. Feature analysis for correlation studies of simultaneous EEG-fMRI data: A proof of concept for neurofeedback approaches. Conf Proc IEEE Eng Med Biol Soc. 2015; 4065-8.

[17] Wang,Y.,Sokhadze, E.M.,El-Baz, A.S.,Li, X.,Sears, L.,Casanova, M.F.,Tasman, A. Relative Power of Specific EEG Bands and Their Ratios during Neurofeedback Training in Children with Autism Spectrum Disorder. Front Hum Neurosci. 2016, 14(9):723.

[18]Herman,S.T., Abend, N.S., Bleck, T.P., Chapman, K.E., Drislane, F.W., Emerson, R.G., Gerard, E.E., Hahn, C.D., Husain, A.M, Kaplan P.W., LaRoche, S.M., Nuwer, M.R., Quigg, M., Riviello, J.J., Schmitt, S.E., Simmons, L.A., Tsuchida, T.N., Hirsch, L.J. Consensus statement on continuous EEG in critically ill adults and children, part II: personnel, technical specifications, and clinical practice.J Clin Neurophysiol. 2015; 32(2):96108.

[19] Gruber, T., Keil, A., Müller, M.M. Modulation of induced gamma band responses and phase synchrony in a paired associate learning task in the human EEG.Neurosci. Lett.2001;316: 29-32.

[20]Kober, S.E., Schweige, D., Witte, M., Reichert, J.L., Grieshofer, P., Neuper, C., Wood G. (2015). Specific effects of EEG based neurofeedback training on memory functions in post-stroke victims. J Neuroeng Rehabil, 2015, 1(12): 107.

[21] Gruzelier, J.,Egner,T. Critical validation studies of neurofeedback.Child Adolesc Psychiatr Clin N Am.2005, 14(1): 83-104.

[22] Engelbregt, H.J., Keeser, D., van Eijk, L., Suiker, EM., Eichhorn, D., Karch, S., Deijen, J.B., Pogarell, O. Short and long-term effects of sham-controlled prefrontal EEG-neurofeedback training in healthy subjects. Clin Neurophysiol. 2016; 127(4):1931-7.

[23] Linden, M., Habib, T., Radojevic, V. A controlled study of the effects of EEG biofeedback on cognition and behavior of children with attention deficit disorder and learning disabilities. Biofeedback Self Regul.1996; 21: 35-49.

[24] Paret, C., Kluetsch, R., Ruf, M., Demirakca, T., Hoesterey, S., Ende, G., Schmahl, C. Down-regulation of amygdala activation with real-time fMRI neurofeedback in a healthy female sample. Front Behav Neurosci. 2014; 18(8): 299.

[25] Yoo, J.J., Hinds, O., Ofen N., Thompson T.W., Whitfield-Gabrieli S., Triantafyllou C., Gabrieli J.D.E. When the brain is prepared to learn: enhancing human learning using real-time fMRI. NeuroImage. 2012;59:846-852.

[26]Zotev, V., Yuan, H., Misaki, M., Phillips, R., Young, K.D., Feldner, M.T., Bodurka, J. Correlation between amygdala BOLD activity and frontal EEG asymmetry during real-time fMRI neurofeedback training in patients with depression. Neuroimage Clin.2016; 12(11): 224-38.

[27]Zotev,V., Krueger, F., Phillips, R., Alvarez, R.P., Simmons, W.K., Bellgowan, P., Drevets, W.C., Bodurka, J. Self-regulation of amygdala activation using real-time FMRI neurofeedback. PLoS One. 2011;6(9):e24522.

[28] Diego, M.A.,Jones, N.A.,Field, T.,Hernandez-Reif, M., Schanberg, S.,Kuhn, C.,McAdam, V.,Galamaga, R.,Galamaga, M. Aromatherapy positively affects mood, EEG patterns of alertness and math computations. Int J Neurosci. 1998; 96(3-4): 217-24.

[29]Henkin, R.I.,Levy,LM. Lateralization of brain activation to imagination and smell of odors using functional magnetic resonance imaging (fMRI): left hemispheric localization of pleasant and right hemispheric localization of unpleasant odors. J Comput Assist Tomogr. 2001;25(4): 493-514.

[30] Sayorwan, W.,Ruangrungsi, N., Piriyapunyporn, T., Hongratanaworakit, T. Kotchabhakdi, N.,Siripornpanich, V. Effects of inhaled rosemary oil on subjective feelings and activities of the nervous system. Sci Pharm. 2013;81(2):531-42.

\section{Volume 5 Issue 7, July 2016 www.ijsr.net}




\section{International Journal of Science and Research (IJSR) \\ ISSN (Online): 2319-7064}

Index Copernicus Value (2013): 6.14 | Impact Factor (2015): 6.391

[31] Lis-Balchin, M., Hart, S. Studies on the mode of action of the essential oil of Lavender (LaParetvandula angustifolia P. Miller) Phytother Res. 1999;13: 540-2.

[32] Kristsidina, M., Newton, T., Asimakopoulu K., The effects of lavender scent on dental patient anxiety levels: a controlled trial cleansing gel effects on relaxation. Comm. Oral Epidemiology. 2010;38: 83-87.

[33] Manley CH. Psychophysiological effect of odor. Crit Rev Food Sci Nutr.1993;33(1): 57-62.

[34] Moskowitz,H.R.,Gerbers,C.L. Functional properties of the olfactory system: psychophysics. Dimensional salience of odors. Ann NY Acad Sci. 1974 ;237(0): 116.

[35]Zald, D.H., Pardo, J.V. Functional neuroimaging of the olfactory system in humans. Int $\mathrm{J}$ Psychophysiol. 2000;36: 165-81.

[36] Micoulaud-Franchi, J.A., McGonigal, A., Lopez, R., Daudet, C., Kotwas, I., Bartolomei, F. Electroencephalographic neurofeedback: Level of evidence in mental and brain disorders and suggestions for good clinical practice. Neurophysiol Clin. 2015; 45(6): 423-33.

[37] Cabaña-Muñoz ME, Parmigiani-Izquierdo JM, BravoGonzález LA, Kyung HM, Merino JJ. Increased Zn/Glutathione Levels and Higher Superoxide Dismutase-1 Activity as Biomarkers of Oxidative Stress in Women with Long-Term Dental Amalgam Fillings: Correlation between Mercury/Aluminium Levels (in Hair) and Antioxidant Systems in Plasma. PLoS One. $2015 ; 15 ; 10(6): \mathrm{e} 0126339$

[38] Cabaña-Muñoz ME, Parmigiani-Izquierdo JM, Parmigiani- Cabaña JM, Merino JJ. Safe Removal of Amalgam Fillings in Dental Clinic: Use of Synergic Nasal Filters (Active Carbon) and Phytonaturals.. International Journal of Science and Research (IJSR) 2015; 4 (3): 2319-7064 ). March

\section{Author Profile}

Dr. María Eugenia Cabaña-Muñoz $\mathrm{Ph}$ Murcia University Universidad de Murcia. Medical College (UM, Spain). Teaching: Odontology field (Master UM) and toxicology of heavy metals, International papers on heavy metals and Odontology. Nutrition and Odontology. Chairman: Congress on Miniscrews, Clinical experience in Odontology field: more than 25 years.

Dr. Carmen Pérez-Laso is Assistant Professor. (Psychobiology Dept. UNED, Madrid). Researcher (Neuroscience field, 19 papers).

Dr. José María Parmigiani-Izquierdo is Ph Student (Murcia University UM, Medical College, Spain). Director of CIROM Clinic (Murcia). Clinical experience in Odontology field: more than 25 years. Teaching: Implantology Master (UM).

International papers on implantology and heavy metals

Dr. José Joaquín Merino did BSch and PhD (Universidad Complutense de Madrid, UCM, Spain). Conway Institute of Molecular Biology (Ireland). Scholar Research (MCP, USA). Teaching and Research: Psychobiology Dept, UNED, Madrid). Biomedical Researcher "Ramon and Cajal Research program" (IdiPaz, Madrid). Researcher: Neuropathology and Neuroinmunoogy and Neuroscience and Regenerative Medicine field. Postdoctoral Researcher (Biochemistry and Molecular Biology Dept; I.U.I.N, UCM. Madrid, Spain).
33 international papers (Impact factor IF: 95, more than 700 citations), 100 Congress. Director of Doctoral Thesis. National research Award. 